ACCEPTED MANUSCRIPT

\title{
Low-cost, 4-system, precise GNSS positioning: a GPS, Galileo, BDS and QZSS ionosphere-weighted RTK analysis
}

To cite this article before publication: Robert Odolinski et al 2017 Meas. Sci. Technol. in press https://doi.org/10.1088/1361-6501/aa92eb

\section{Manuscript version: Accepted Manuscript}

Accepted Manuscript is "the version of the article accepted for publication including all changes made as a result of the peer review process, and which may also include the addition to the article by IOP Publishing of a header, an article ID, a cover sheet and/or an 'Accepted

Manuscript' watermark, but excluding any other editing, typesetting or other changes made by IOP Publishing and/or its licensors"

This Accepted Manuscript is @ 2017 IOP Publishing Ltd.

During the embargo period (the 12 month period from the publication of the Version of Record of this article), the Accepted Manuscript is fully protected by copyright and cannot be reused or reposted elsewhere.

As the Version of Record of this article is going to be / has been published on a subscription basis, this Accepted Manuscript is available for reuse under a CC BY-NC-ND 3.0 licence after the 12 month embargo period.

After the embargo period, everyone is permitted to use copy and redistribute this article for non-commercial purposes only, provided that they adhere to all the terms of the licence https://creativecommons.org/licences/by-nc-nd/3.0

Although reasonable endeavours have been taken to obtain all necessary permissions from third parties to include their copyrighted content within this article, their full citation and copyright line may not be present in this Accepted Manuscript version. Before using any content from this article, please refer to the Version of Record on IOPscience once published for full citation and copyright details, as permissions will likely be required. All third party content is fully copyright protected, unless specifically stated otherwise in the figure caption in the Version of Record.

View the article online for updates and enhancements. 


\title{
Received: date / Accepted: date
}

\author{
Robert Odolinski $^{1}$ - Peter J.G. Teunissen ${ }^{2,3}$
}

\section{Low-cost, 4-system, precise GNSS positioning: a GPS, Galileo, BDS and QZSS ionosphere-weighted RTK analysis}

\begin{abstract}
With the combination of the emerging GNSSs, single-frequency (SF) precise RTK positioning becomes possible. In this contribution we evaluate such low-cost ublox receiver and antenna performance when combining real data of four CDMA systems, namely L1 GPS, E1 Galileo, L1 QZSS, and B1 BDS. Comparisons are made to more expensive dual-frequency (DF) GPS receivers and antennas. The formal and empirical ambiguity success rates and positioning precisions will first be evaluated while making use of $\mathrm{L} 1+\mathrm{E} 1$, so as to investigate whether instantaneous SF RTK is possible without the need of B1 BDS or L1 QZSS. This follows by an analysis of the SF 4-system model performance when the residual ionosphere can be ignored and modeled as a function of the baseline length, respectively. The analyses are conducted for a location in Dunedin, New Zealand, and compared to Perth, Australia with the better visibility of BDS and QZSS. The results indicate that successful instantaneous precise RTK positioning is feasible while using L1 GPS and E1 Galileo data, and that the SF 4-system model is competitive to DF GPS even when residual ionospheric delays are present. We finally demonstrate that when the impact from the ionosphere increases and more than one epoch is needed for successful ambiguity resolution, the SF 4-system model performance can still remain competitive to the DF GPS receivers. This is particularly true in Perth with more satellites and when higher than customary elevation cut-off angles need to be used to avoid low-elevation multipath.
\end{abstract}

Keywords Low-cost receiver, Multi-GNSS, Real Time Kinematic (RTK), ionosphere-weighted model

\section{R. Odolinski}

Robert.Odolinski@otago.ac.nz

P. J. G. Teunissen

P.Teunissen@ curtin.edu.au

${ }^{1}$ National School of Surveying, University of Otago, Dunedin, New Zealand

${ }^{2}$ Department of Spatial Sciences, GNSS Research Centre, Curtin University of Technology, Perth, Australia

${ }^{3}$ Department of Geoscience and Remote Sensing, Delft University of Technology, Delft, The Netherlands

\section{Introduction}

The regional Chinese BeiDou Navigation Satellite System (BDS) became operational in 2011 and consists to date (May 2017) of 14 satellites. The first Japanese QZSS satellite was launched in 2010, and a further 16 European Galileo and 32 GPS satellites are now available for positioning. The combination of the Global Navigation Satellite Systems (GNSSs) makes single-frequency (SF) real-time kinematic (RTK) positioning possible (Verhagen et al, 2012; He et al, 2014; Teunissen et al, 2014; Zhao et al, 2014; Odolinski et al, 2015a).

Combined GPS+Galileo RTK positioning was investigated in (Julien et al, 2003; Odijk and Teunissen, 2013; Paziewski and Wielgosz, 2015), and some first results using BDS outside of China are reported in (Montenbruck et al, 2013; Nadarajah et al, 2013). SF GPS and GLONASS Precise Point Positioning (PPP) results were shown in (de Bakker and Tiberius, 2017), and combined GPS, GLONASS, BDS and Galileo PPP in (Lou et al, 2016; Pan et al, 2017). With the development of low-cost (a few hundred USDs) multiGNSS SF RTK receivers, competitive ambiguity resolution and millimeter-level positioning performance can potentially be obtained in comparison to more expensive (thousands USDs) dual-frequency (DF) GPS receivers (Odolinski and Teunissen, 2016, 2017).

A range of positioning applications can be further enhanced by using multi-constellation low-cost RTK receivers. For example, the significant reduction in cost would be beneficial for precise aircraft navigation and harbour entry by ships (Misra and Enge, 2006), tsunami early warning systems (Schone et al, 2011), earth deformation measurements (Hamling, I. J. et al., 2017), array-based satellite phase bias sensing systems for PPP-RTK (Khodabandeh and Teunissen, 2014), GNSS and inertial navigation system (INS) integration (Wang and Wenbo, 2016), unmanned aerial vehicle (UAV) applications (Mongredien et al, 2016), precise car lane keeping (Knoop et al, 2017), and precision farming (Marucci et al, 2017), to name a few. Other studies on SF GPS RTK using low-cost receivers can be found in 
(Wirola et al, 2006; Takasu and Yasuda, 2008; Wisniewski et al, 2013; Pesyna et al, 2014).

In this contribution we will thus, for the first time, analyse low-cost SF receiver RTK performance using the Code Division Multiple Access (CDMA) signals of L1 GPS, L1 QZSS, E1 Galileo and B1 BDS that are tracked by ublox receivers and antennas. Performance comparisons will be made for a location in Dunedin, New Zealand and Perth, Australia that has a better visibility of BDS and QZSS. All SF results will be assessed against the RTK performance when using more expensive DF (L1, L2) GPS receivers and antennas.

This contribution is organized as follows. In Sect. 2 the multi-GNSS and standalone GPS RTK model is described, and in Sect. 3 the GNSS data and stochastic model are presented. The combined SF L1+E1 GPS+Galileo model is then evaluated in Sect. 4 by formal and empirical integer ambiguity success-rates and positioning precisions. This is done so as to assess whether instantaneous lowcost precise RTK positioning is currently possible without the need of regional BDS or QZSS. In Sect. 5 the SF 4-system RTK model is then analysed in Dunedin (NZ) and Perth (AU), respectively. In Sect. 6 the corresponding performance for a $8.9 \mathrm{~km}$ baseline is evaluated when residual slant ionospheric delays become present, and the ionosphere-weighted model needs to be used. In Sect. 7 the formal ambiguity success-rates for longer baselines, with more impact from the ionosphere, are evaluated, and we end up with a summary and conclusions in Sect. 8.

\section{Multi-GNSS single-frequency RTK model}

In this section, we describe the single-baseline single-frequency (SF) RTK model. We assume for now that the two receivers track all GNSSs on the same frequency $j$. When the baseline length between the receivers is sufficiently short, say a few kilometers, the satellite orbit errors and relative slant ionospheric delays can be neglected in the model, which is also referred to as the "ionosphere-fixed" model. The receivers are assumed to be from the same manufacturer (receiver make, type and firmware), which thus allows us to assume that the inter-system biases (ISBs) between all constellations are zero (Odijk and Teunissen, 2013; Odolinski et al, 2015a). We can then use inter-system doubledifferencing (DD) with respect to a common reference satellite (Julien et al, 2003) and thus maximize the redundancy of the model.

The full-rank, ionosphere-fixed, SF instantaneous (singleepoch) DD observation equations of systems $A$ and $B$ read

$$
\begin{aligned}
E\left[\begin{array}{l}
D_{m}^{T} \phi \\
D_{m}^{T} p
\end{array}\right] & =\left[\begin{array}{cc}
\lambda I_{m-1} & D_{m}^{T} G \\
0 & D_{m}^{T} G
\end{array}\right]\left[\begin{array}{l}
a \\
b
\end{array}\right] \\
\mathrm{D}\left[\begin{array}{c}
D_{m}^{T} \phi \\
D_{m}^{T} p
\end{array}\right] & =\left[\begin{array}{cc}
D_{m}^{T} Q_{\phi \phi} D_{m} & 0 \\
0 & D_{m}^{T} Q_{p p} D_{m}
\end{array}\right]
\end{aligned}
$$

in which $\mathrm{E}[$.$] denotes the expectation and \mathrm{D}[$.] the dispersion operator, respectively, $\phi=\left[\phi_{A}^{T}, \phi_{B}^{T}\right]^{T}$ is the combined phase vector, $p=\left[p_{A}^{T}, p_{B}^{T}\right]^{T}$ the combined code vector, $a=$

$\left[a_{A}^{T}, a_{B}^{T}\right]^{T}$ the combined inter-system DD integer ambiguity vector of size $(m-1)$, with $m=m_{A}+m_{B}$ the total number of satellites for systems $A$ and $B$, and $b$ is the baseline increment vector of size 3 (size 4 if a Zenith Tropospheric Delay (ZTD) is included). The matrix $D_{m}^{T}=\left[-e_{m-1}, I_{m-1}\right]$ is the differencing operator, where $e_{m-1}$ is the $(m-1)$ vector of ones, $I_{m-1}$ the identity matrix of dimension $(m-1)$, $\lambda I_{m-1}$ is the wavelength matrix, and $G=\left[G_{A}^{T}, G_{B}^{T}\right]^{T}$ contains the undifferenced line-of-sight unit-vectors and has a dimension of $m \times 3$. The $\phi_{*}$ and $p_{*}$ vectors of size $m_{*}$ contain the between-receiver single-differenced phase and code observables, respectively, for systems $*=\{A, B\}$, where $A$ can be GPS and $B$ Galileo, BDS and QZSS respectively. The variance matrices $Q_{\phi \phi}=\operatorname{blkdiag}\left(Q_{\phi_{A} \phi_{A}}, Q_{\phi_{B} \phi_{B}}\right)$ and $Q_{p p}=\operatorname{blkdiag}\left(Q_{p_{A} p_{A}}, Q_{p_{B} p_{B}}\right)$, respectively, contains

$Q_{\phi_{*} \phi_{*}}=2 \times \sigma_{\phi_{j_{*}}}^{2} W_{*}^{-1}$

$Q_{p_{*} p_{*}}=2 \times \sigma_{p_{j_{*}}}^{2} W_{*}^{-1}$

with zenith-referenced phase $\sigma_{\phi_{j_{*}}}^{2}$ and code $\sigma_{p_{j_{*}}}^{2}$ variances. The matrix $W_{*}=\operatorname{diag}\left(w^{1}, \ldots, w^{m_{*}}\right)$ contains the satellite $s_{*}=1_{*}, \ldots, m_{*}$ elevation-dependent weight (Euler and Goad, 1991), given as follows,

$w^{s_{*}}=\left[1+10 \exp \left(-\frac{\theta^{s_{*}}}{10}\right)\right]^{-2}$

where $\theta^{s_{*}}$ is the elevation of satellite $s_{*}$ in degrees. The notation 'diag' denotes a diagonal and 'blkdiag' a block diagonal matrix, respectively. Note that the B1 frequency of regional BDS does not overlap the $\mathrm{L} 1$ and $\mathrm{E} 1$ frequencies, so that classical system-specific differencing is employed

To deal with baselines when residual ionospheric delays become present, we will use the so called "ionosphereweighted" model. We then add a vector with slant ionosphere pseudo-observations $\imath=\left[\boldsymbol{l}_{A}^{T}, \boldsymbol{l}_{B}^{T}\right]^{T}$ to the model in (1), and parameterize the ionospheric delays. By including these observations they can provide us with stochastic information of the delays between stations, see further Teunissen (1998b); Odijk (2002).

The redundancy and solvability condition of the DD DF single-system, with $m_{*}$ satellites, and the SF multi-GNSS model (1), with $m=m_{A}+m_{B}$, is given as follows,

$$
\begin{array}{lll}
\text { DF Single-system } & \text { redundancy: } & 2\left(m_{*}-1\right)-3[-1] \\
& \text { solvability: } & m_{*} \geq 4[+1] \\
\text { SF Multi-GNSS } & \text { redundancy: } & m_{A}+m_{B}-4[-1] \\
& \text { solvability: } & m_{A}+m_{B} \geq 4[+1]
\end{array}
$$

From Equation (4) it follows that four satellites are needed to solve the model, and five if a ZTD is also included which represents the square brackets. This implies that the multiGNSS model is solvable even when less than four (or five) satellites are in view for each system, whereas it would not suffice when one uses a single-system. 

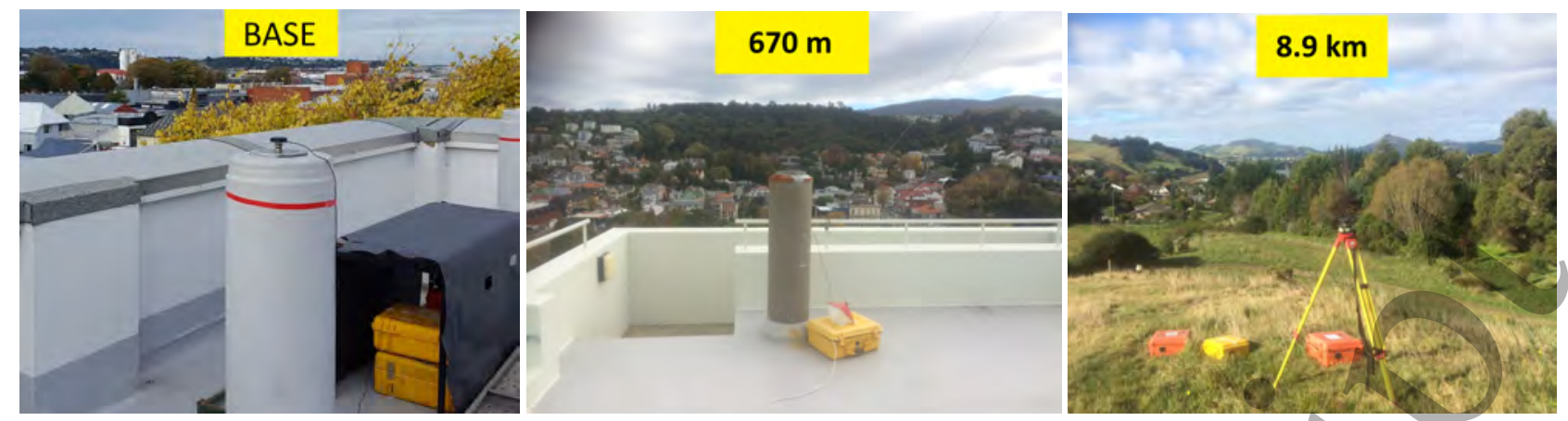

(a) Dunedin receiver setups.

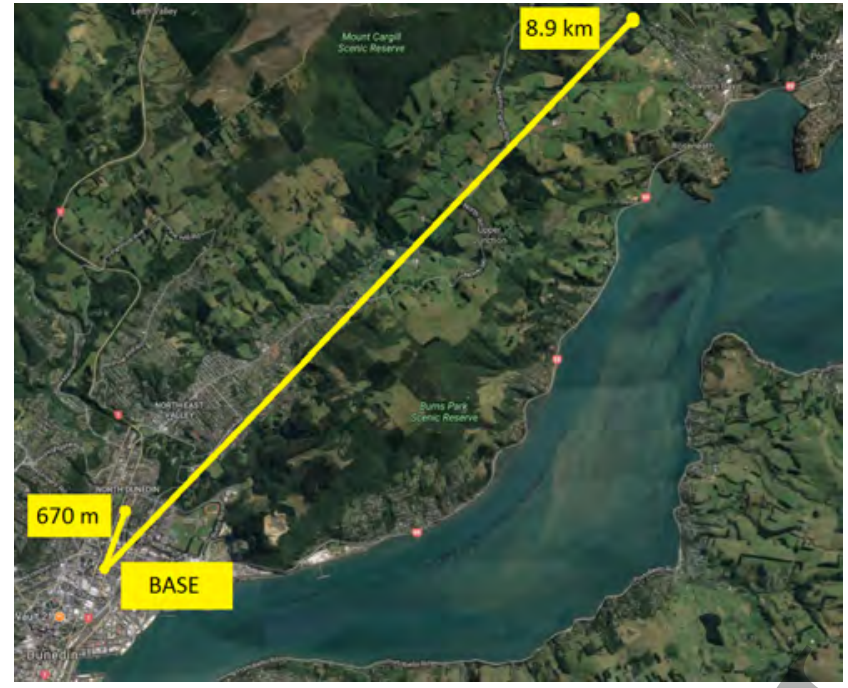

(b) Dunedin baselines.

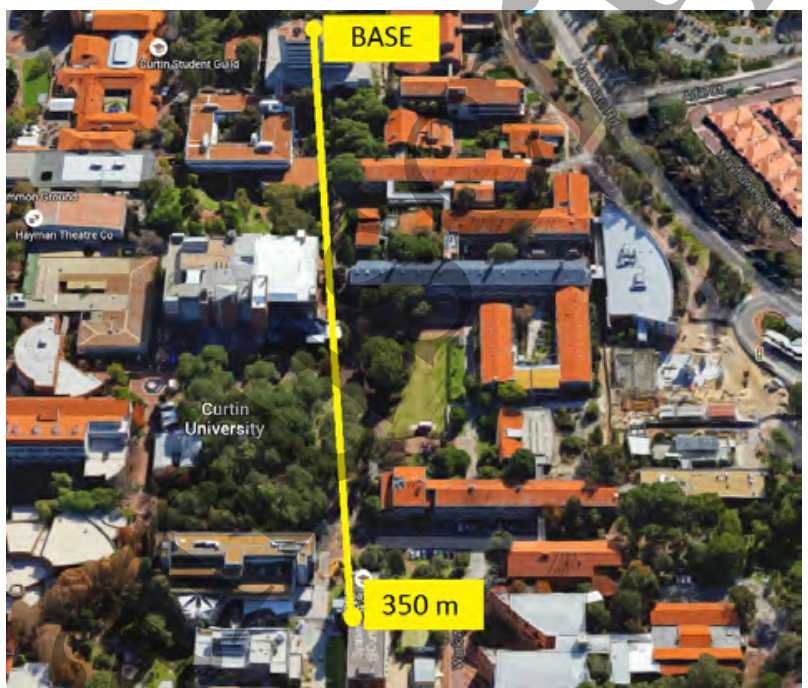

(c) Perth baseline.

Fig. 1 Dunedin: GNSS ublox EVK-M8T receivers collecting data for SF 4-system single-baseline RTK. a Patch and Trimble Zephyr 2 antennas are connected to the receivers that collect data in May 5-7 and June 2-3, 2017. Trimble 5700 DF GPS receivers have been connected to the same Zephyr 2 antennas as well so as to truly track the same GPS constellation. b The baselines formed to evaluate the RTK performance in Dunedin are $670 \mathrm{~m}$ and $8.9 \mathrm{~km}$, respectively. Perth: c Two days in Jan 20-21, 2017 are also used to evaluate the performance where the ublox EVK-M8T receivers are connected to Trimble chokering (TRM59800.00) antennas to form a $350 \mathrm{~m}$ baseline. The maps were obtained through Map data (c) Google.

\section{Australia and New Zealand experiment setups, and stochastic model}

The receivers used to collect single-frequency (SF, L1 GPS, L1 QZSS, E1 Galileo, B1 BDS) and dual-frequency (DF, GPS L1+L2) data are depicted in Figure 1. A measurement interval of $30 \mathrm{~s}$ was employed to avoid any receiverinduced time-correlation (Odolinski and Teunissen, 2017), and the Detection, Identification and Adaptation (DIA) procedure by Teunissen (1990a) was used to detect and identify outliers. The LAMBDA method (Teunissen, 1995) was moreover used for ambiguity resolution. The ionosphereweighted model (Teunissen, 1998b; Odijk, 2002) was employed for the $8.9 \mathrm{~km}$ baseline in Dunedin, where ionosphere pseudo-observations were included and the slant ionospheric delays parameterized.

Figure 2 depicts the number of satellites tracked by a receiver in Perth (AU) and Dunedin (NZ), respectively. The figure illustrates that the number of satellites when combining all four systems (black lines) does not go below eighteen in Perth and fourteen in Dunedin, respectively. The number of Galileo satellites is also always two or more over the entire day for both locations. Finally one can see that the QZSS satellite is visible over a longer time period and the number of regional BDS satellites is larger in Perth when compared to Dunedin. This implies that the 4-system model strength will be better in Perth, which can be measured by its redundancy (c.f. Odolinski et al (2015a)). We can thus expect to have a better ambiguity resolution and positioning performance in Perth (Odolinski and Teunissen, 2016).

The least-squares variance component (LS-VCE) procedure (Teunissen, 1988; Teunissen and Amiri-Simkooei, 2008) was used to determine the zenith-referenced and undifferenced standard deviations (STDs) for code and phase, as depicted in Table 1.

The model used for LS-VCE is further described in (Odolinski and Teunissen, 2017), and all STDs were estimated based on data independent of the data to be analysed. The zenith-referenced and double-differenced (DD) slant ionospheric delay STD $\sigma_{1}^{2}$ that is used in the stochastic model for the $8.9 \mathrm{~km}$ baseline was determined from data one day before the data periods that are analysed. This STD was empirically estimated by taking the mean of the STDs of all slant ionospheric delays over the entire observation time- 
Table 1 Zenith-referenced and undifferenced code and phase STDs for Perth (AU) and Dunedin (NZ) on frequency $j_{*}=1_{*}, \ldots, f_{*}$ for system *. The STDs have been estimated based on models including satellites from all 4-systems and $24 \mathrm{~h}$ of data (30 s) independent from the data to be used for positioning (c.f. Table 2). The DD STD for the ionosphere pseudo observations and the $8.9 \mathrm{~km}$ baseline was estimated to $\hat{\sigma}_{l}=5$ mm based on independent data.

\begin{tabular}{|c|c|c|c|c|c|c|}
\hline Location (baseline length) & Date (hh:mm:ss) & Receiver/antenna & System & Frequency & $\hat{\sigma}_{p_{j_{*}}}[\mathrm{~cm}]$ & $\hat{\sigma}_{\phi_{j_{*}}}[\mathrm{~mm}]$ \\
\hline \multirow[t]{4}{*}{ Perth $(350 \mathrm{~m})$} & Jan 20, 2017, & ublox EVK-M8T & GPS & L1 & 46 & 2 \\
\hline & 00:00:00 UTC & +TRM59800.00 & BDS & B1 & 36 & 2 \\
\hline & & & Galileo & E1 & 37 & 2 \\
\hline & & & QZSS & L1 & 43 & 2 \\
\hline \multirow[t]{4}{*}{ Dunedin $(670 \mathrm{~m})$} & May 5, 2017, & ublox EVK-M8T & GPS & L1 & 43 & 2 \\
\hline & 00:10:30 UTC & + patch & BDS & B1 & 56 & \\
\hline & & & Galileo & E1 & 36 & \\
\hline & & & QZSS & L1 & 50 & \\
\hline \multirow[t]{4}{*}{ Dunedin $(670 \mathrm{~m})$} & June 2, 2017, & ublox EVK-M8T & GPS & L1 & 42 & 2 \\
\hline & 22:18:30 UTC & +Zephyr & BDS & B1 & 41 & 2 \\
\hline & & & Galileo & E1 & & 2 \\
\hline & & & QZSS & L1 & 46 & 2 \\
\hline \multirow[t]{2}{*}{ Dunedin $(670 \mathrm{~m})$} & June 2, 2017, & Trimble 5700 & GPS & L1 & 18 & 2 \\
\hline & 22:18:30 UTC & +Zephyr & & L2 & & 2 \\
\hline
\end{tabular}

Table 2 Positioning models and $24 \mathrm{~h}$ of data $(30 \mathrm{~s})$ to be analysed (Figure 1) with stochastic model based on the VCE results in Table 1 , where different combinations of the systems will be compared

\begin{tabular}{|c|c|c|c|c|c|}
\hline Experiment & Location (baseline length) & Date (hh:mm:ss) & Receiver/antenna & Systems & Frequencies \\
\hline \multirow[t]{2}{*}{ Section 4 and 5} & Dunedin, NZ (670 m) & May 6, 2017, & ublox EVK-M8T & GPS+QZSS & $\mathrm{L} 1+\mathrm{L} 1$ \\
\hline & & 00:10:30 UTC & + patch & +Galileo+BDS & $+\mathrm{E} 1+\mathrm{B} 1$ \\
\hline \multirow[t]{2}{*}{ Section 4 and 5} & Dunedin, NZ (670 m) & June 3, 2017, & ublox EVK-M8T & GPS+QZSS & $\mathrm{L} 1+\mathrm{L} 1$ \\
\hline & & 22:18:30 UTC & +Zephyr & + Galileo+BDS & $+\mathrm{E} 1+\mathrm{B} 1$ \\
\hline \multirow[t]{2}{*}{ Section 4 and 5} & Dunedin, NZ (670 m) & June 3, 2017 & Trimble 5700 & GPS & $\mathrm{L} 1+\mathrm{L} 2$ \\
\hline & & 22:18:30 UTC & +Zephyr & & \\
\hline \multirow[t]{2}{*}{ Section 5 and 7} & Perth, AU (350 m) & Jan 21, 2017, & ublox EVK-M8T & GPS+QZSS & $\mathrm{L} 1+\mathrm{L} 1$ \\
\hline & & 00:00:00 UTC & +TRM59800.00 & +Galileo+BDS & $+\mathrm{E} 1+\mathrm{B} 1$ \\
\hline \multirow[t]{2}{*}{ Section 6 and 7} & Dunedin, NZ (8.9 km) & May 6, 2017, & ublox EVK-M8T & GPS+QZSS & $\mathrm{L} 1+\mathrm{L} 1$ \\
\hline & & $00: 10: 30$ UTC & & + Galileo+BDS & $+\mathrm{E} 1+\mathrm{B} 1$ \\
\hline Section 6 and 7 & Dunedin, NZ (8.9 km) & $\begin{array}{l}\text { May 7, } 2017, \\
\text { 01:40:00 UTC }\end{array}$ & $\begin{array}{l}\text { Trimble } 5700 \\
+ \text { Zephyr }\end{array}$ & GPS & $\mathrm{L} 1+\mathrm{L} 2$ \\
\hline
\end{tabular}

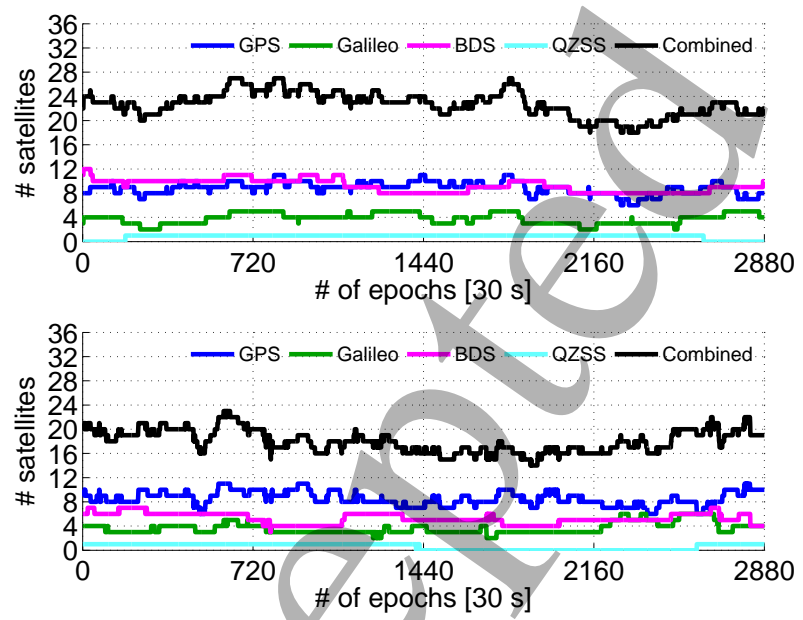

Fig. 2 Number (\#) of satellites for an elevation cut-off angle of $10^{\circ}$ and the receivers in Figure 1 during 24 h, with Perth (top) in Jan 21 and Dunedin (bottom) in May 6, 2017.

span. The Australian Space Forecast Centre did not report on any increased geomagnetic disturbance during this period, which implies that the ionospheric conditions were indeed relatively calm.

The STDs in Table 1 are overall smaller when using Trimble Zephyr 2 antennas due to their better multipath suppression and signal reception in comparison to the ublox low-cost patch antennas (Pesyna et al, 2014). For example the B1 code STD decreases from $56 \mathrm{~cm}$ (May 5) for patch antennas to $41 \mathrm{~cm}$ (June 2) when Zephyr antennas are used for the same receiver-setup. The larger B1 STD value is believed to be caused by multipath since large fluctuations were seen in the patch-antenna DD B1 residuals that repeat between two days (May 5 and May 6). We can also see a difference between the code STDs in Perth in comparison to Dunedin due to site-specific environmental effects such as multipath, and that different types of antennas are used.

In the following Sections we will investigate the positioning performance based on the data depicted in Table 2, which is independent of the data that was used to determine the stochastic model in Table 1.

\section{Low-cost GPS+Galileo RTK positioning}

We begin by analyzing the current SF L1+E1 GPS+Galileo RTK model, since it will allow us to assess whether lowcost precise RTK positioning is possible using the global constellations without the need of regional BDS or QZSS. We will compare the SF low-cost ublox receiver performance to that of DF GPS when using Trimble 5700 re- 
ceivers. The evaluations will be given for the $670 \mathrm{~m}$ baseline in Dunedin as depicted in Table 2 and Figure 1.

We start investigating the ambiguity resolution performance by considering the ambiguity bootstrapped (BS) successrate (SR). We make use of the following formula by Teunissen (1998a),

$\mathrm{P}\left[\check{\mathrm{z}}_{\mathrm{IB}}=z\right]=\prod_{i=1}^{n}\left[2 \Phi\left(\frac{1}{2 \sigma_{\hat{z}_{i \mid I}}}\right)-1\right]$

where $\mathrm{P}\left[\check{z}_{\mathrm{IB}}=z\right]$ denotes the probability of correct integer estimation of the integer bootstrapped (IB) estimator $\check{z}_{\mathrm{IB}}$ and $\sigma_{\hat{z}_{i \mid}}, i=1, \ldots, n, I=\{1, \ldots,(i-1)\}$, denote the conditional STDs of the LAMBDA decorrelated ambiguities. The BS SR (5) is easy to compute and a sharp lower bound of the integer least-squares (ILS) SR (Teunissen, 1999).

Figure 3 depicts the single-epoch (instantaneous) BS SRs for $\mathrm{L} 1+\mathrm{E} 1$ and $\mathrm{L} 1+\mathrm{L} 2$, respectively, as a function of the elevation cut-off angles. These SRs were obtained by averaging over $24 \mathrm{~h}$ and conditioned on Positional Dilution of Precisions (PDOPs) $\leq 10$ to obtain $P_{s \mid \mathrm{PDOP}<10}$. By excluding epochs with large PDOPs we can ensure that the fixed position solutions have millimeter-centimeter level precisions (Teunissen et al, 2014; Odolinski and Teunissen, 2016). Large PDOPs above ten occur when the receiversatellite geometry is poor due to similar line-of-sight unitvectors, for example when all satellites lie on the surface of a cone (Teunissen, 1990b; Zaminpardaz et al, 2017).

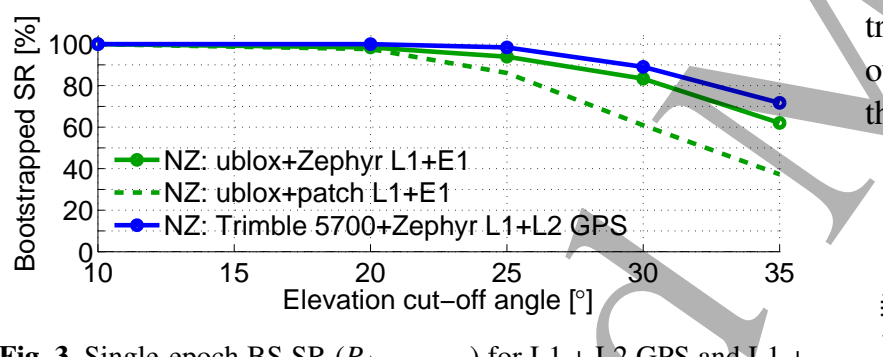

Fig. 3 Single-epoch BS SR $\left(P_{s \mid \mathrm{PDOP} \leq 10}\right)$ for L1 + L2 GPS and L1 + $\mathrm{E} 1 \mathrm{GPS}+$ Galileo (ISBs-fixed) models as function of the elevation cut-off angle, based on the $670 \mathrm{~m}$ baseline data as depicted in Table 2. The BS SRs are taken as a mean of all single-epoch SRs over one day, and conditioned on PDOP $\leq 10$

Although the results in Figure 3 look nice, they only hold true for PDOP less than or equal to ten. This implies that if this condition is rarely met, the given nice results are also rarely available. To investigate this PDOP availability further we depict in Figure 4 the number of epochs over all positioning epochs that have the $\mathrm{PDOP} \leq 10$ condition fulfilled, for the different models and elevation cutoff angles. In this figure one can see that this condition is always fulfilled when GPS is combined with the current Galileo constellation (green lines) for cut-off angles up to $30^{\circ}$. Whereas the DF GPS model (dark blue lines) only fulfills this condition for cut-off angles below $25^{\circ}$. When Galileo will be of full constellation by 2020 (GalileoICD, 2014), we predict that this PDOP-condition will always be met when the dual-system combination is used also for the cut-off angle of $35^{\circ}$.

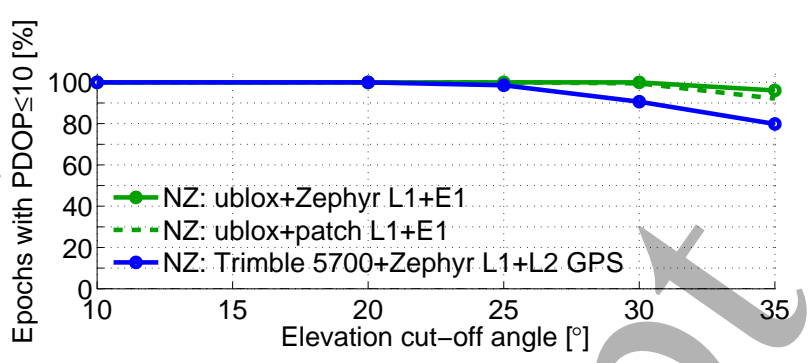

Fig. 4 Availability over $24 \mathrm{~h}$ of PDOP $\leq 10$ for L1 + L2 GPS and L1 + E1 GPS + Galileo (ISBs-fixed) models as function of the elevation cut-off angle, based on the $670 \mathrm{~m}$ baseline data in Table 2

We can also see in Figure 3 that the SRs decrease with increasing cut-off angles, and that the Zephyr/antenna model (full green lines) are then having larger SRs than the corresponding patch-antenna model (dotted green lines). This is mainly due to the difference in Galileo constellation between the two models, since these satellites do not repeat their positions on a day to day basis but rather every tenth sidereal day (GabileoICD, 2014), as well as the (slightly) better L1 code precision when using the Zephyr antenna (c.f. Table 1). To illustrate this further we give in Figure 5 the corresponding number of tracked Galileo satellites in green for an elevation cut-off angle of $10^{\circ}$, together with the number of satellites being four denoted by dotted red lines. In this figure we can see that the Zephyr data in June 3 indeed has a larger number of Galileo satellites over the day in comparison to the patch data collected 28 days earlier in May 6, 2017. For instance the patch data (top row) tracks more than three Galileo satellites $55 \%$ of the time over the day, whereas the Zephyr data (bottom row) does this $78 \%$ of the time.
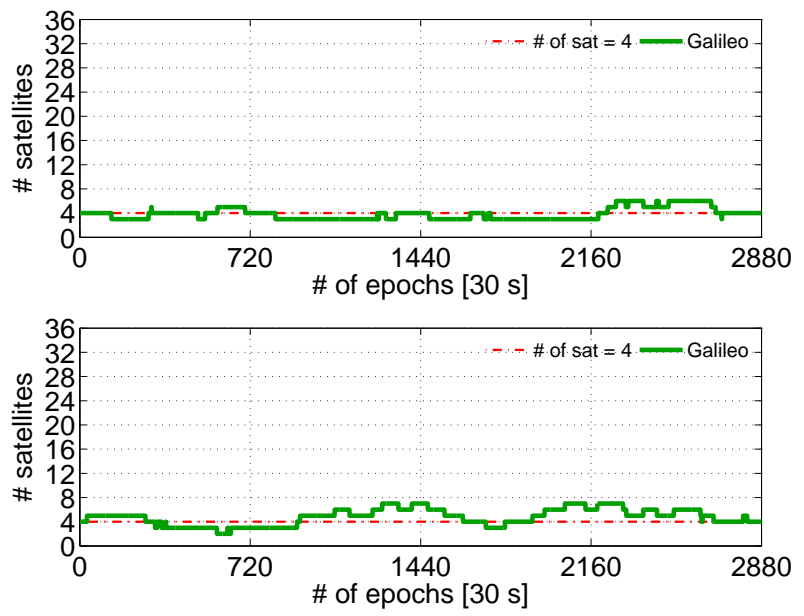

Fig. 5 Number (\#) of Galileo satellites (four denoted by red lines) for an elevation cut-off angle of $10^{\circ}$ during $24 \mathrm{~h}$, with ublox+patch (top) in May 6 and ublox+Zephyr data (bottom) in June 3, 2017.

If we inspect the excellent ambiguity resolution performance results in Figure 3 again, it shows that the ubloxreceiver SF GPS+Galileo models provide for competitive instantaneous ambiguity resolution performance to the DF GPS model for cut-off angles up to $20^{\circ}$. For instance, the models achieve the excellent performance of $99.9 \%$ and 

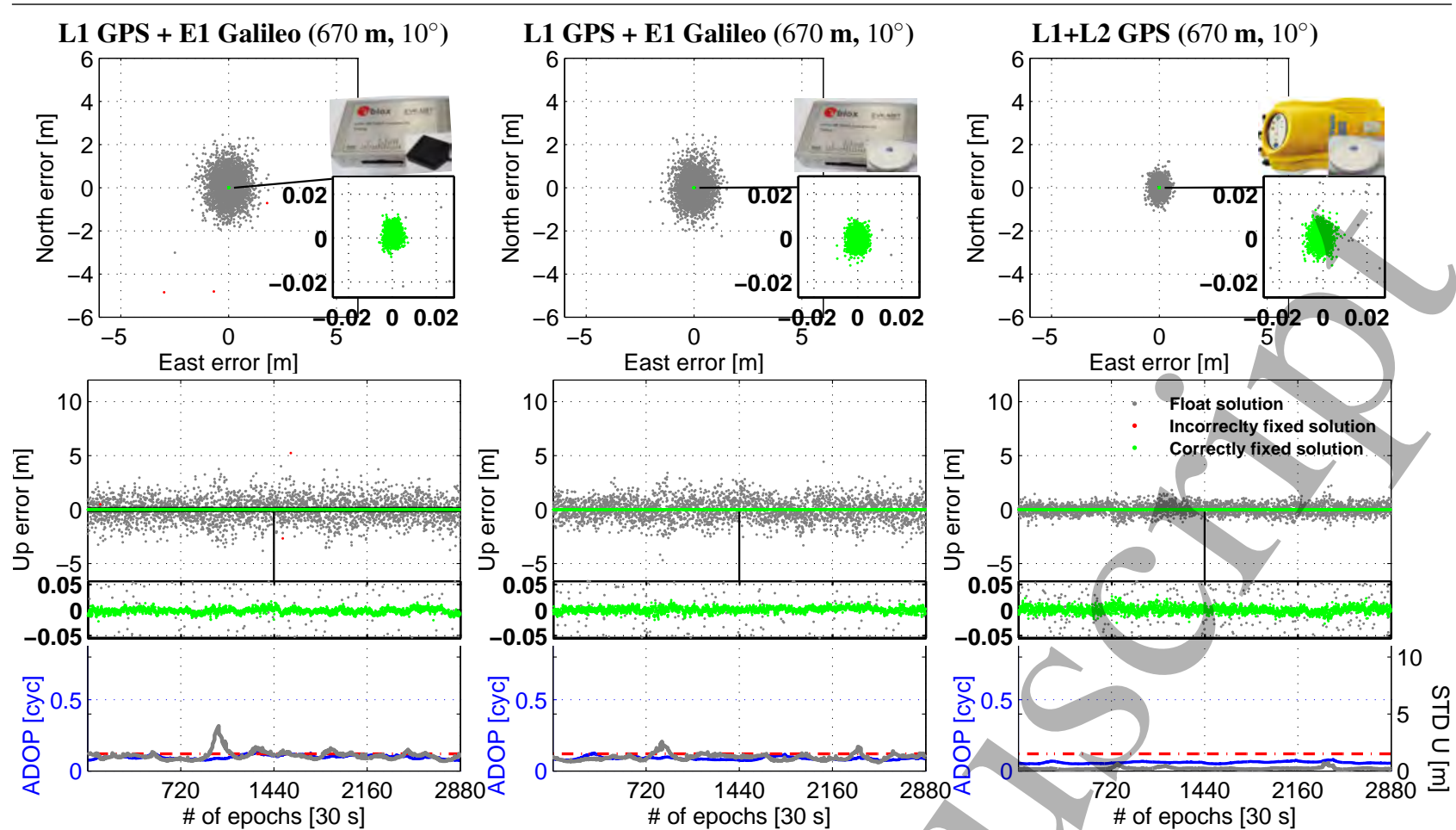

Fig. 6 Horizontal (N, E) scatterplots and vertical (U) time series in Dunedin for a $670 \mathrm{~m}$ baseline and L1 + E1 GPS+Galileo ublox+patch (1st column) with $99.9 \%$ ILS SR $\left(P_{S_{E}}\right), \mathrm{L} 1+\mathrm{E} 1$ ublox+Zephyr (2nd column) with 100\% ILS SR, and Trimble 5700 L1+L2 GPS (3rd column) with $100 \%$ ILS SR, using $10^{\circ}$ cut-off angle. The $24 \mathrm{~h}$ data periods used are depicted in Table 2 . The correctly and incorrectly fixed positioning solutions are depicted as green and red dots, respectively, and ambiguity-float solutions as gray dots. Below the vertical time-series the ADOP is depicted in dark blue color, the 0.12 cycles level as red, and ambiguity-float Up formal STDs are shown in gray

$100 \%$ BS SRs for the cut-off angle of $10^{\circ}$, while connected to patch and Zephyr antennas, respectively. The nice results in Figure 3 thus implies that successful, instantaneous precise positioning is potentially feasible with low-cost SF RTK receivers and the global Galileo and GPS constellations, and without the need of regional BDS or QZSS.

To verify the formal claims in Figure 3 we depict in Figure 6 the corresponding instantaneous horizontal (N/E) and vertical $(\mathrm{U})$ positioning errors, when compared to very precise benchmark coordinates. These benchmark coordinates were obtained through an ambiguity-fixed multi-epoch model, while assuming the ambiguities as time-constant parameters over the entire time span so that their uncertainty can be neglected. Zoom-in windows are also given so as to show that the correctly fixed solutions (green dots) have about a two-order of magnitude better precision, at the millim centimeter-level, than the ambiguity-float (gray) and incorrectly fixed (red dots) counterparts. The first column depicts $\mathrm{L} 1+\mathrm{E} 1 \mathrm{ublox}+$ patch, second column the corresponding Zephyr-antenna model, and the third column the Trimble 5700 L1+L2 GPS model. The correctly fixed solutions were determined by comparing the epoch-by-epoch solutions to a set of reference ambiguities. These reference ambiguities were also obtained by assuming the ambiguities as time-constant in a dynamic model over the entire observation time-span. Below the vertical time-series we depict the Ambiguity Dilution of Precision (ADOP, see Teunissen (1997)), and the ambiguity-float Up formal STDs so as to reflect their consistency with the empirical positioning results. The 0.12 cycle level is also depicted since when the ADOP falls below this level, then as a rule-of-thumb one can expect an ambiguity SR larger than $99.9 \%$ (Odijk and Teunissen, 2008). The empirical ILS SR is given as,

$P_{s_{E}}=\frac{\# \text { of correctly fixed epochs }}{\text { total \# of epochs }}$

Note that any inconsistencies that can be seen in the excursions for the Up STDs between the SF models in the left two columns of Figure 6 are due to the Galileo satellites that do not repeat between the two days (c.f. Figure 5 ), which results in different receiver-satellite geometries for certain epochs when combined with GPS (c.f. Figure 4).

Figure 6 shows that the ADOPs stay below or near the 0.12 cycle level for all models, with excellent ILS SRs of $99.9 \%$ and $100 \%$ when using patch and Zephyr antennas, respectively. The ILS SRs are also in good agreement With the BS SRs in Figure 3, which implies realistic LSVCE STDs used in the stochastic model (Table 1). We have thus empirically illustrated that successful low-cost instantaneous L1+E1 RTK positioning is feasible, and that the performance is competitive to the DF GPS model (which has an ILS SR of $100 \%$ ). This competitive performance is similar to what one can achieve when L1+B1 GPS+BDS is used in Perth and Dunedin, respectively, see the analyses in (Teunissen et al, 2014; Odolinski and Teunissen, 2016, 2017).

\section{Low-cost 4-system RTK positioning}

In this section we add the regional BDS and QZSS to our SF GPS+Galileo models to investigate the corresponding 
RTK performance that is achievable in Perth (AU) and Dunedin angles, the ILS SRs are computed as follows, (NZ), respectively. By doing so we can evaluate how much better such performance would be in Perth when compared to Dunedin as an effect of the better visibility of the regional BDS and QZSS.

$$
P_{s_{E} \mid \mathrm{PDOP} \leq 10}=\frac{\# \text { of correctly fixed epochs and } \mathrm{PDOP} \leq 10}{\text { total } \# \text { of epochs }}
$$

Figure 7 depicts the single-epoch SRs that are averaged over $24 \mathrm{~h}$ of data and conditioned on PDOPs $\leq 10$. The SF 4-system model in Perth (black lines) has an excellent performance with $100 \%$ SRs for all cut-off angles, which clearly exceeds the SRs of the DF GPS model (dark blue lines) for cut-off angles above $20^{\circ}$. One can also see an improvement of the Dunedin SF 4-system model performance when Zephyr antennas are used (red lines).

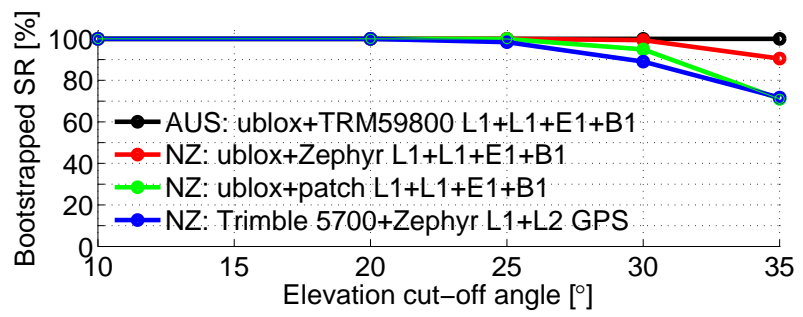

Fig. 7 Single-epoch BS SR $\left(P_{s \mid \mathrm{PDOP}<10}\right)$ for $\mathrm{L} 1+\mathrm{L} 2$ GPS and 4system L1 + L1 + E1 + B1 (ISBs-fixed) models as function of the elevation cut-off angle, based on the $350 \mathrm{~m}$ and $670 \mathrm{~m}$ baseline data as depicted in Table 2. The BS SRs are taken as a mean of all singleepoch SRs over one day, and conditioned on PDOP $\leq 10$

Most interestingly one can see in Figure 7 that the ublox+patch SF 4-system model (green lines) has a similar instantaneous ambiguity resolution performance to the DF GPS model for cut-off angles up to $35^{\circ}$ in Dunedin. This can also be compared to the corresponding L1+B1 GPS+BDS model that achieves such performance only for a cut-off angle of $10^{\circ}$ (Odolinski and Teunissen, 2017). We have thus formally shown that the low-cost SF 4-system model with patch antennas can give competitive ambiguity resolution performance to the DF GPS model for higher than customary elevation cut-off angles. This is of importance in areas such as urban canyons or when low-elevation multipath is present and to be avoided.

In Figure 8 the positioning results corresponding to Figure 7 are shown. This is given for cut-off angles of $25^{\circ}$ at the top three rows and $35^{\circ}$ at the bottom three rows, where PDOPs are depicted in cyan color so as to reflect the receiver-satellite geometry strength. The DF GPS model in the right column is based on data from Trimble 5700 receivers in Dunedin, and its performance is expected to be similar to that of Perth. In addition, any correctly fixed solutions with large PDOPs $>10$ are depicted as red triangles, which in general also have ADOPs $\leq 0.12$ cycles. This is done to illustrate that good ambiguity resolution performance does not always mean a similarly good positioning performance (c.f. Teunissen et al (2014); Odolinski and Teunissen (2016); Zaminpardaz et al (2017)). Since we now have some PDOPs above ten for these higher cut-off
By making use of equation (7) we can thus make sure that the ILS SRs then directly correspond to the precise correctly fixed solutions as depicted in green color.

Figure 8 shows that the DF GPS model in the right column has instances where the ambiguities are correctly fixed with ADOPs below or close to the 0.12 cycle level but the PDOPs are at the same time above 10, which consequently leads to positioning scatters at the decimetermeter level as denoted by the red triangles. This is particularly pronounced for the cut-off angle of $35^{\circ}$ at bottom. When investigating some of these instances with very large PDOPs, it turned out to indeed be epochs with satellites that formed a cone-like geometry similar to Figure 6 in (Zaminpardaz et al, 2017). One can also see gaps in the GPS-only positioning time-series and PDOPs at the bottom three rows, which is due to the number of GPS satellites not being above or equal to four so one can solve the model (4). However, all correctly fixed solutions with PDOPs below 10 have a precision of millimeter-centimeter level as depicted by the zoom-ins.

Figure 8 shows, as also predicted in Figure 7, that the SF 4-system model in Perth, in the middle column, obtains an excellent ambiguity resolution and positioning performance, where none of the correctly fixed solutions have PDOPs above 10 for both cut-off angles. There is however a slight increase in PDOPs between epoch 1440 and 2160 for the cut-off angle of $35^{\circ}$, due to instances of setting satellites below the cut-off angle during this particular time-period, which results in slightly larger corresponding vertical positioning errors. The SF 4-system model in Dunedin has also more incorrectly fixed solutions when the ADOPs exceed the 0.12 cycle level, for instance just before epoch 720 and around epoch 1440 for this higher cut-off angle.

Most importantly Figure 8 confirms empirically the excellent formal results in Figure 7, namely that the SF 4system model in Dunedin, in the left column, have larger PDOP-conditioned ILS SRs than the DF GPS model for both cut-off angles.

\section{Low-cost 4-system RTK positioning for a 8.9 km baseline}

In this section we will evaluate the corresponding lowcost SF 4-system RTK performance in Dunedin for a 8.9 $\mathrm{km}$ baseline when the residual ionospheric delays are estimated in an ionosphere-weighted model, with the stochastic model settings in Table 1 . In the next section we will investigate the corresponding formal ambiguity resolution performance for baseline lengths longer than $8.9 \mathrm{~km}$.

Figure 9 shows the low-cost SF 4-system and DF GPS positioning results for cut-off angles of $10^{\circ}$ and $30^{\circ}$ at the 

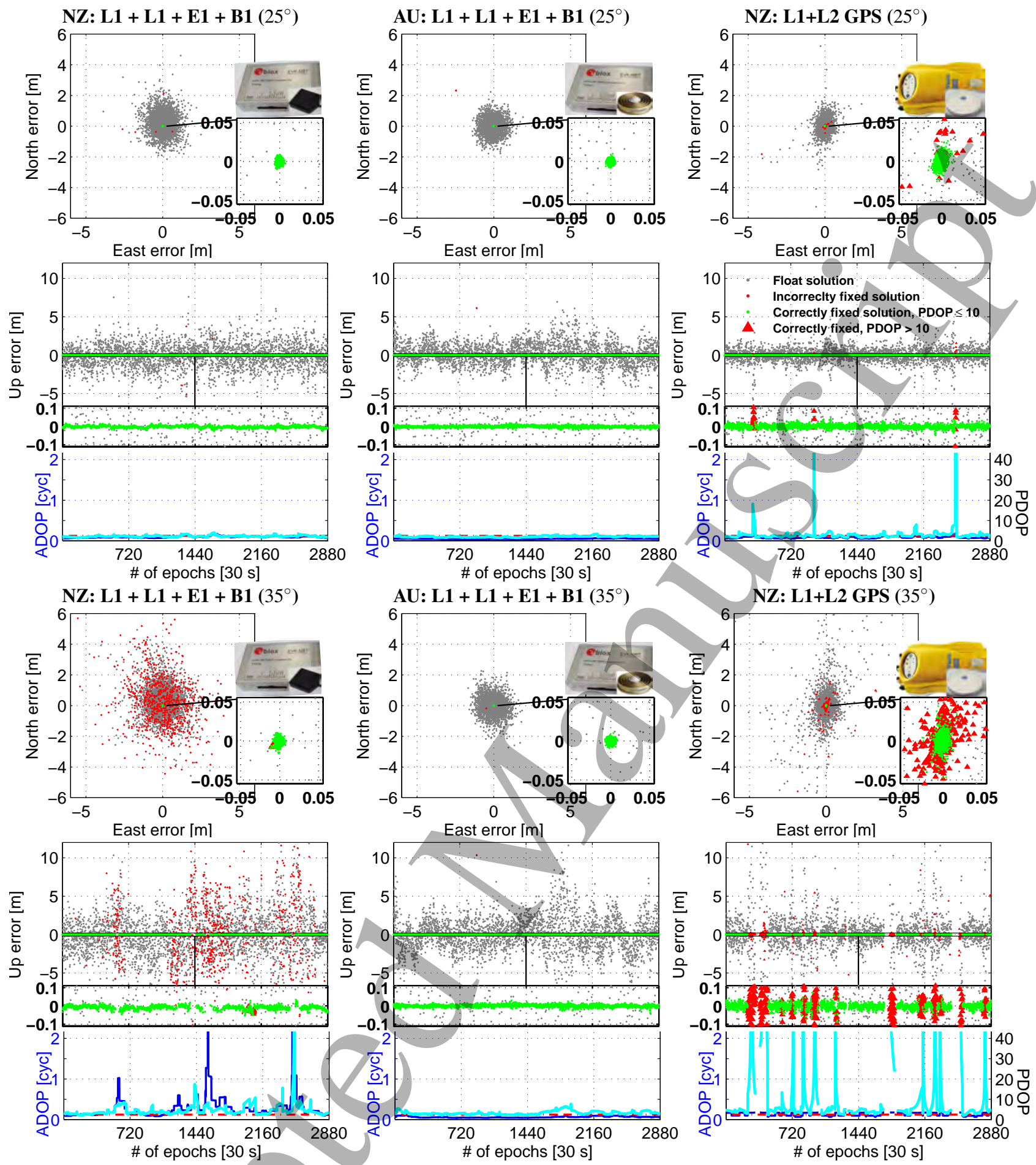

Fig. 8 Horizontal (N, E) scatterplots and vertical (U) time series in Dunedin for a $670 \mathrm{~m}$ baseline and in Perth for a $350 \mathrm{~m}$ baseline. The 4 system L1 + L1 + E1 + B1 ublox+patch in Dunedin (1st column) achieves 99.8\% (72.3\%) ILS SR, for the cut-off angle of $25^{\circ}$ (top three rows) and $35^{\circ}$ (bottom three rows), respectively. The 4-system L1 + L1 + E1 + B1 ublox+TRM59800.00 in Perth (2nd column) has 100\% (100\%) ILS SR, and Trimble 5700 L1+L2 GPS in Dunedin (3rd column) has 98.5\% (71.7\%) ILS SR. The $24 \mathrm{~h}$ data periods used are depicted in Table 2. The incorrectly fixed positioning solutions are depicted as red dots, and ambiguity-float solutions as gray dots. The correctly fixed solutions are depicted as green dots when PDOP $\leq 10$, and red triangles when PDOP $>10$. Below the vertical time-series the ADOP is depicted in dark blue color, the 0.12 cycles level as red, and PDOPs are shown in cyan. The ILS SRs $\left(P_{S_{E} \mid \mathrm{PDOP} \leq 10}\right)$ are taken as a mean of all single-epochs over one day, and conditioned on PDOP $\leq 10$ so that they only represent the precise correctly fixed solutions in green color 
Low-cost, 4-system, precise GNSS positioning: a GPS, Galileo, BDS and QZSS ionosphere-weighted RTK analysis
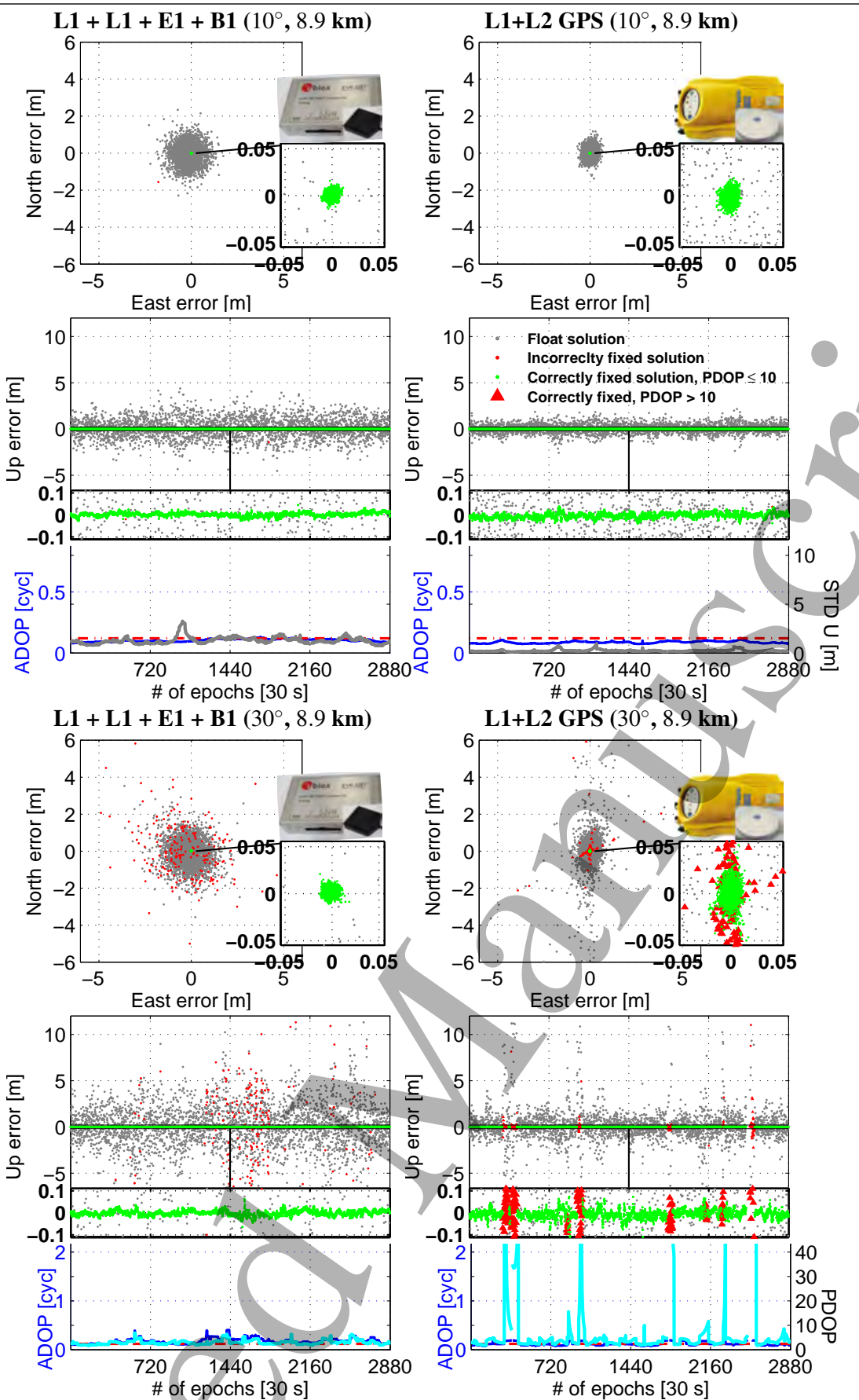

Fig. 9 Horizontal (N, E) scatterplots and vertical (U) time series in Dunedin for a $8.9 \mathrm{~km}$ baseline and 4-system L1 + L1 + E1 + B1 ublox+patch (1st column) with $100 \%\left(92.7 \%\right.$ ) ILS SR, for the cut-off angle of $10^{\circ}$ (top three rows) and $30^{\circ}$ (bottom three rows), respectively. The Trimble $5700 \mathrm{~L} 1+\mathrm{L} 2$ GPS model (2nd column) has $100 \%$ (88.5\%) ILS SR, respectively. The 24 h data periods used are depicted in Table 2 . The incorrectly fixed positioning solutions are depicted as red dots, and ambiguity-float solutions as gray dots. The correctly fixed solutions are depicted as green dots when PDOP $\leq 10$, and red triangles when PDOP $>10$. Below the vertical time-series the ADOP is depicted in dark blue color, the 0.12 cycles level as red, ambiguity-float Up formal STDs are shown in gray at top, and PDOPs in cyan at bottom. The ILS SRs $\left(P_{s_{E} \mid \mathrm{PDOP} \leq 10}\right)$ are taken as a mean of all single-epochs over one day, and conditioned on PDOP $\leq 10$ so that they only represent the precise correctly fixed solutions in green color

top three and bottom three rows, respectively. This is given together with the ADOPs and formal Up STDs at top, and PDOPs at the bottom row.

Figure 9 shows that the 4-system model with patch antennas obtains an excellent $100 \%$ ILS SR performance for the cut-off angle of $10^{\circ}$. This results in a corresponding $100 \%$ availability of precise fixed solutions similar to DF GPS since none of the models obtain PDOPs larger than ten. Most importantly the ublox model obtains a PDOPconditioned ILS SR of about $93 \%$ for the cut-off angle of $30^{\circ}$. This can be compared to the DF GPS model that has a corresponding ILS SR of about $88 \%$ due to the many instances with PDOPs above 10 that results in correspondingly poorer positioning scatters (red triangles).

Table 3 summarizes the $8.9 \mathrm{~km}$ baseline positioning results for different combination of the 4-systems, where STDs are given in local North, East and Up positioning errors as computed by comparing the estimated positions to the very precise benchmark coordinates. Below each ILS SR the STDs and ILS SRs are computed based on PDOP $\leq 10$ 
when different from their unconditioned counterparts, and within brackets the corresponding BS SRs are also given so as to reflect consistency between the empirical and formal results.

Table 3 shows that the fixed solutions improve from decimeter-meter-level down to millimeter-level when the $\mathrm{PDOP} \leq 10$ condition is applied for the cut-off angles of $30^{\circ}-35^{\circ}$ and the DF GPS model (c.f. Figure 9). One can also see in the table a comparable ILS SR performance for the GPS+BDS and GPS+Galileo SF models for all cutoff angles, for instance the ILS SR is about $98 \%$ for both models when using an elevation cut-off angle of $10^{\circ}$. However, the GPS+Galileo model gets slightly better for cut-off angles higher than $25^{\circ}$ as the number of satellites become then more similar to the GPS+BDS model, and the Galileo E1 code observations are also more precise than the B1 BDS counterparts (c.f. Table 1). Finally Table 3 shows that the SF 4-system model obtains competitive (PDOP-condition ILS SRs to DF GPS for all cut-off angles.

In conclusion we have thus illustrated that the low-cost SF 4-system model with patch antennas can give competitive ambiguity resolution and positioning performance to the DF GPS model also for a baseline length of $8.9 \mathrm{~km}$ for various elevation cut-off angles.

\section{Low-cost RTK performance for several ionosphere-weighted baselines}

The ionospheric conditions for the baseline analysed in the previous section were relatively quiet with a DD slant ionospheric delay STD of about $\sigma_{t}=0.6 \mathrm{~mm} / \mathrm{km}$ used in the ionosphere-weighted stochastic model (c.f. Table 1). Therefore in this section/we will compare the formal BS SR performance to that of more active ionospheric conditions such as the ones experienced in (Odolinski and Teunissen, 2017), where the DD slant ionospheric delay STD was about $\sigma_{l}=1.4 \mathrm{~mm} / \mathrm{km}$ that also follows the rule-ofthumb by Schaffrin and Bock (1988). Comparisons will be made between low-cost SF 4-system RTK in Dunedin (NZ) and Perth (AU), and the DF GPS and SF L1+E1 GPS+Galileo performance will be evaluated as well. The relative ZTD is now parameterized as well, a condition PDOP $\leq 10$ is applied and the success-rates are computed by averaging over all epochs. For these computations we make use of the same code and phase precisions as depicted in Table 1 and satellite constellations from the data depicted in Table 2, i.e. Jan 21 in 2017 for Perth and May 6 and May 7 in 2017 for Dunedin. Note that for the following analysis we only need the design matrix and the stochastic model, i.e., real data is not necessary.

Figure 10 depicts the BS SRs for the two different $\sigma_{l}$ settings and four different positioning models for an elevation cut-off angle of $10^{\circ}$. Since it is of interest to investigate how long baselines that are allowed for instantaneous or very fast precise positioning solutions, we compute the BS SRs as a function of baseline lengths ranging from $8.9 \mathrm{~km}$ to $20 \mathrm{~km}$ using the settings of $\sigma_{l}$ above. In all evaluations we aim at instantaneous (1-epoch, at top) or fast ambiguity resolution (2- and 4-epochs at middle and bottom rows, respectively). In the multi-epoch models we use a dynamic model for the ambiguities and relative ZTD where they are treated as time-constant parameters over the specific timespan. The Kalman filter is initialized at the first epoch and dafter each time-window of 2 or 4 epochs have passed the filter is re-initialized at the second epoch, and the whole procedure is repeated again.

As expected the SRs in Figure 10 decrease with increasing baseline lengths and $\sigma_{l}$, and DF observations become more important (Odijk, 2002; Odolinski et al, 2015b). In the DF case the slant ionospheric delays are shared between the two frequencies that thus leads to more precise corresponding estimates than in the SF case, which in turn affects the ambiguity variance matrix $Q_{\hat{a} \hat{a}}$ and consequently the ambiguity resolution performance (c.f. Equation 5.54 at p. 123 in (Odijk, 2002)).

In the left column of Figure 10 we can see that the SF 4-system models achieve an excellent instantaneous ambiguity resolution performance when the ionospheric conditions are relatively calm, where the performance in Perth (black lines) is competitive with DF GPS (in dark blue on top of the black lines) for all the analysed baseline lengths. The corresponding Dunedin performance (red lines) is then competitive with DF GPS for baseline lengths up to about $10 \mathrm{~km}$. The SF L1+E1 GPS+Galileo model (green lines), however, has a poor instantaneous performance since the ZTD is now also included. When two epochs are included, in the left column and second row, the performance for the Dunedin SF 4-system model becomes competitive to DF GPS for all baselines, whereas four epochs are needed for the L1+E1 model to obtain similar performance.

The situation becomes different, however, when the ionospheric conditions are more active, as depicted in the right 

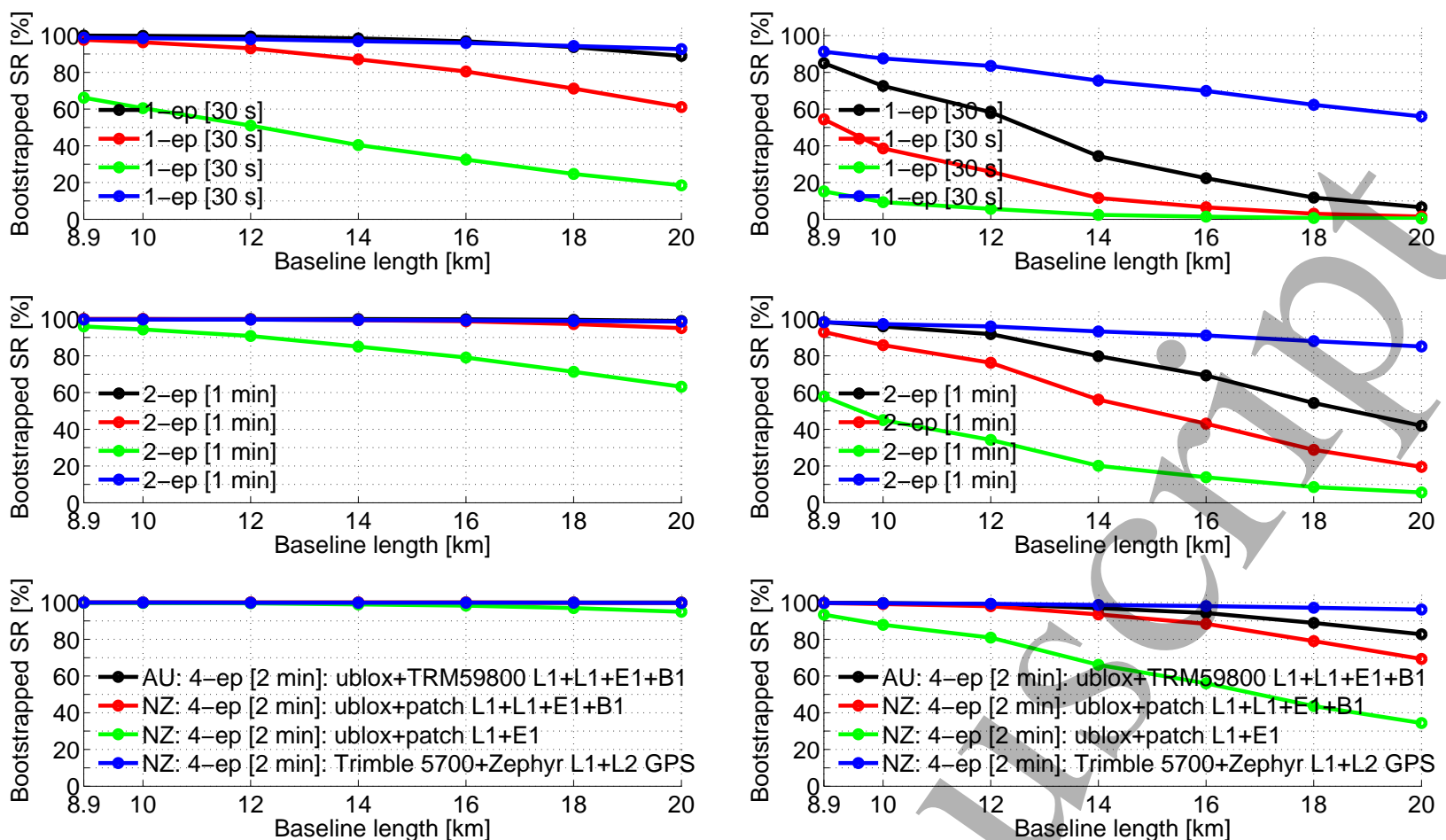

(a) $\sigma_{l}=\mathbf{0 . 6} \mathbf{~ m m} / \mathbf{k m}$ : DF GPS vs SF $\mathrm{L} 1+\mathrm{E} 1$ and $\mathrm{L} 1+\mathrm{L} 1+\mathrm{E} 1+\mathrm{B} 1$

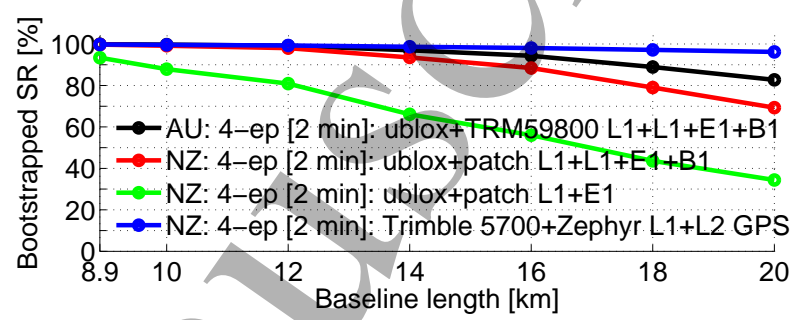

Fig. 10 Cut-off $10^{\circ}$ : BS SRs $\left(P_{s}\right)$ with $\sigma_{l}=0.6 \mathrm{~mm} / \mathrm{km}($ left $)$ and $\sigma_{l}=1.4 \mathrm{~mm} / \mathrm{km}($ right $)$ for $\mathrm{L} 1+\mathrm{L} 2 \mathrm{GPS}$, SF L1+E1 and 4-system L1 + $\mathrm{L} 1+\mathrm{E} 1+\mathrm{B} 1$ (ISBs-fixed) in Dunedin and Perth as function of the baseline length. The \# of epochs (ep) is 1, 2 and 4 epochs from the top to bottom rows, respectively. The BS SRs are taken as a mean of all SRs over one day, and the relative ZTD is parameterized in the models.
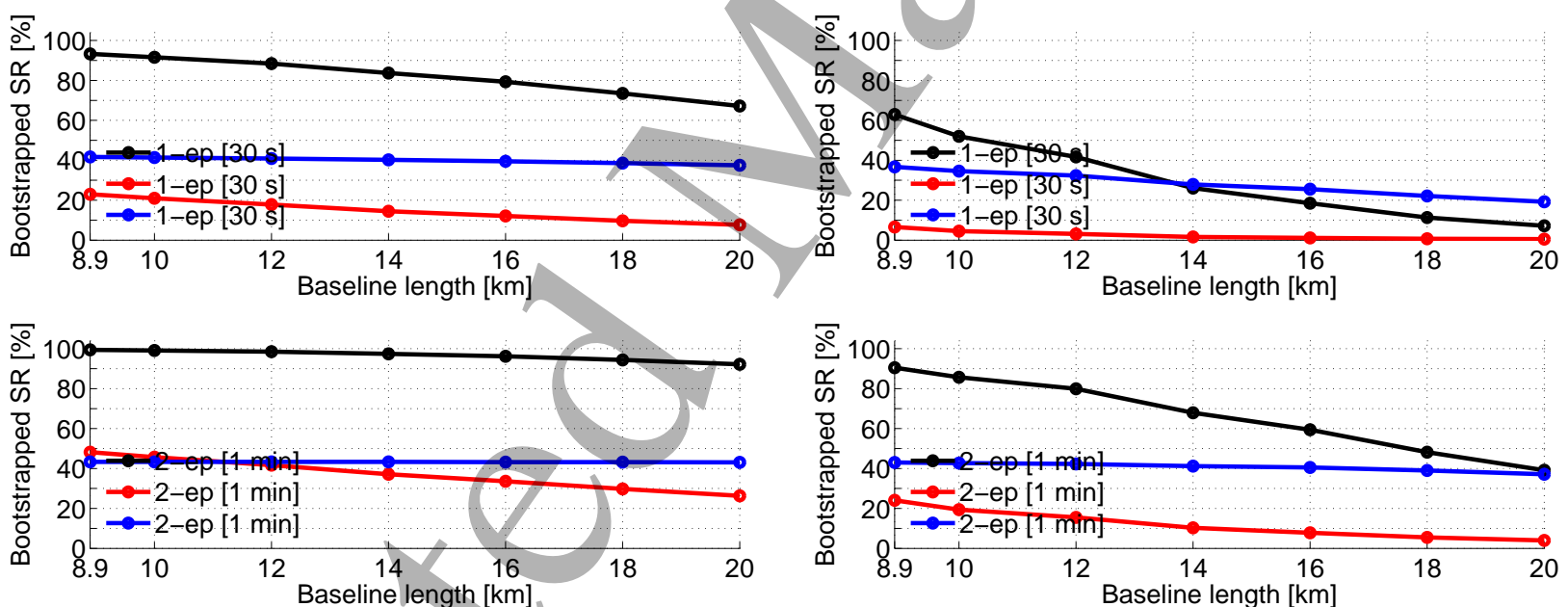

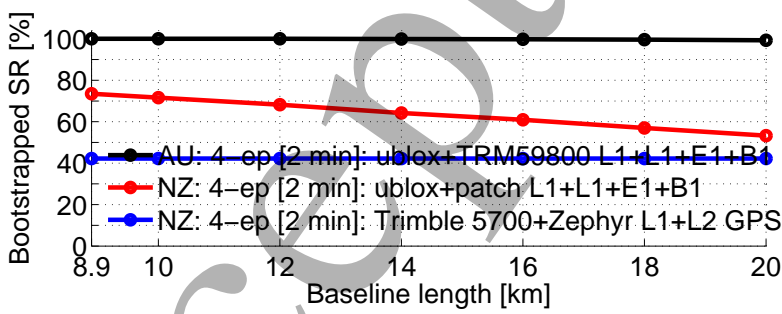

(a) $\sigma_{l}=\mathbf{0 . 6} \mathbf{~ m m} / \mathbf{k m}$ : DF GPS vs SF L1+L1+E1+B1

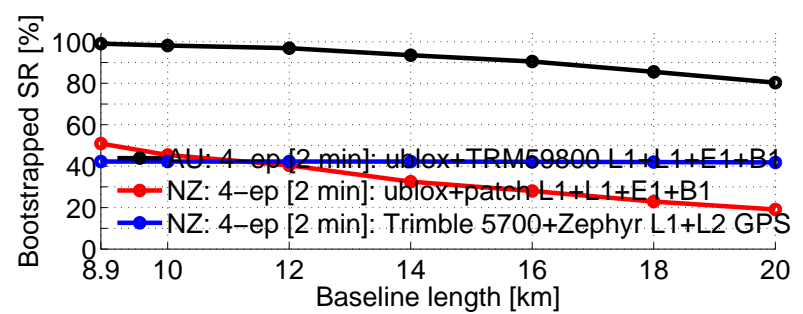

(b) $\sigma_{l}=\mathbf{1 . 4} \mathbf{~ m m} / \mathbf{k m}$ : DF GPS vs SF L1+L1+E1+B1

Fig. 11 Cut-off $35^{\circ}$ : BS SRs $\left(P_{s \mid \mathrm{PDOP} \leq 10}\right)$ with $\sigma_{l}=0.6 \mathrm{~mm} / \mathrm{km}($ left $)$ and $\sigma_{l}=1.4 \mathrm{~mm} / \mathrm{km}($ right $)$ for L1 $+\mathrm{L} 2 \mathrm{GPS}$ and 4 -system L1 + L1 $+\mathrm{E} 1+\mathrm{B} 1$ (ISBs-fixed) in Dunedin and Perth as function of the baseline length. The \# of epochs (ep) is 1, 2 and 4 epochs from the top to bottom rows, respectively. The BS SRs are taken as a mean of all SRs over one day and conditioned on PDOP $\leq 10$, and the relative ZTD is parameterized in the models. 
column of Figure 10. Here we can see that none of the models can achieve successful instantaneous ambiguity resolution for any baseline, which is consistent with the results in (Odijk, 2002; Odolinski et al, 2015b), and more than one epoch is needed. When two epochs are included, in the right column and second row, the SF 4-system model in Perth has then a competitive ambiguity resolution performance to DF GPS for baseline lengths up to $12 \mathrm{~km}$. The corresponding Dunedin model achieves similar good performance when four epochs are included in the bottom row, and the SF 4-system model in Perth is then competitive to DF GPS for baselines up to $16 \mathrm{~km}$.

Figure 11 depicts the corresponding results for the SF 4-systems and DF GPS RTK when we make use of a much higher elevation cut-off angle of $35^{\circ}$. The DF GPS performance is now, as expected, much worse in comparison to the results in Figure 10, which is mainly due to the fewer number of satellites to solve the model (4). Since a ZTD is included a minimum of five satellites are needed and this results in many epochs during the day where the positions cannot be estimated, which is approximately $56 \%$ of the time over the day. The SF 4-system models with larger number of tracked satellites, however, do not experience similar problems except for a few instances in Dunedin, with approximately $2 \%$ of the epochs over the day when the model cannot be solved.

Most importantly we can thus see in Figure 11 that the SF 4-system model in Perth then has much better overall performance than DF GPS for all baselines when the ionospheric conditions are relatively calm in the left column. The corresponding Dunedin model, however, needs more than one epoch to obtain a competitive or better performance to DF GPS. As expected the instantaneous ambiguity resolution performance degrades significantly when the ionosphere is more active in the right column of Figure 11. Note here that the 4-system model in Perth (black lines) has higher instantaneous SRs than DF GPS (blue lines) for baselines from $8.9 \mathrm{~km}$ to $12 \mathrm{~km}$, which is again due to the lack of positioning availability for the latter model. The DF GPS model can namely only be solved about $44 \%$ of the time over the day for this cut-off angle so that when the SRs are averaged over all epochs they become lower than the 4-system model in Perth for these baselines.

Finally the right column of Figure 11 shows that the SF 4-system model in Perth then gets better SRs than DF GPS for all baseline lengths when more than one epoch is included (second and third row, respectively). This thus illustrates that the low-cost SF 4-system RTK model (in Perth) can potentially achieve an overall better fast ambiguity resolution performance than DF GPS when higher than customary elevation cut-off angles need to be used, and the residual ionospheric delays and ZTD are present.

\section{Conclusions}

In this contribution we analysed the single-frequency (SF) low-cost RTK receiver performance with current (May 2017) L1 GPS, E1 Galileo, B1 BDS and L1 QZSS. The data was collected with low-cost patch and high-grade geodetic antennas. The SF RTK performance was compared to dualfrequency (DF) L1 + L2 GPS while using high-grade receivers and antennas. The performance was evaluated and compared, both formally and empirically, between a location in Perth, Australia and Dunedin, New Zealand, for a few hundred meters and a $8.9 \mathrm{~km}$ baseline, respectively. Formal success-rate evaluations were also conducted for longer baselines, so as to illustrate the degradation in the RTK performance due to the increased presence of residual slant ionospheric delays. It was shown that the low-cost SF receiver RTK solutions have the potential to achieve competitive ambiguity resolution and positioning performance to DF GPS even when small residual ionospheric delays are present, and higher than customary elevation cut-off angles are used.

We demonstrated, both formally and empirically, that successful, instantaneous SF L1+E1 GPS+Galileo RTK positioning is feasible when the residual ionospheric delays can be neglected, and without the need to use the regional BDS or QZSS. By adding B1 BDS and L1 QZSS to SF GPS-Galileo, we also investigated the impact from having a better visibility of the regional BDS and QZSS in Perth and in comparison to Dunedin. We found that successful instantaneous ambiguity resolution is possible for the SF 4-system model in Perth, while still making use of cut-off angles up to $35^{\circ}$. It was also shown that the ublox+patch SF 4-system model in Dunedin can achieve competitive precise positioning performance to DF GPS for higher than customary cut-off angles and a baseline length of $8.9 \mathrm{~km}$, owing to the improved receiver-satellite geometry strength by combining the 4-systems.

Our formal success-rate analysis revealed that DF observations and/or more than one epoch of data are needed when the impact from residual ionospheric delays increases and a relative ZTD is included. Fortunately, however, the SF 4-system models in Dunedin and Perth achieved a more competitive ambiguity resolution performance to DF GPS when more epochs were included, with a better performance in Perth. It was finally shown that such SF 4-system performance in Perth can overall potentially be even better than DF GPS when high elevation cut-off angles are used, which is mainly due to the many epochs where the single-system model cannot be solved since at least five satellites are then needed. With the future full constellations of Galileo, BDS and QZSS, we expect that the models will get stronger so that such combined SF performance will be further improved.

Acknowledgements Dr. Mohammad Choudhury at the GNSS Research Centre of Curtin University provided for the ublox data in Perth. Yong Chien Zheng, Luke McDonald, Julian Thom and Ray McLennan at School of Surveying of University of Otago collected the ublox data in Dunedin. The second author is the recipient of an Australian Research Council (ARC) Federation Fellowship (project number FF0883188). All this support is gratefully acknowledged. 


\section{References}

de Bakker PF, Tiberius CCJM (2017) Real-time multiGNSS single-frequency precise point positioning. GPS Solut doi:10.1007/s10291-017-0653-2

Euler HJ, Goad C (1991) On optimal filtering of GPS dual frequency observations without using orbit information. Bull Geod 65:130-143

GalileoICD (2014) European GNSS (Galileo) Open Service, Signal in Space Interface Control Document, European Union. Tech. rep., 64 pages

Hamling, I J et al (2017) Complex multifault rupture during the $2016 \mathrm{Mw} 7.8$ Kaikoura earthquake, New Zealand. Science doi:10.1126/science.aam7194

He H, Li J, Yang Y, Xu J, Guo H, Wang A (2014) Performance assessment of single- and dual-frequency BeiDou/GPS single-epoch kinematic positioning. GPS Solut 18(3):393-403, doi:10.1007/s10291-013-0339-3

Julien O, Alves P, Cannon ME, Zhang W (2003) A tightly coupled GPS/GALILEO combination for improved ambiguity resolution. In: ENC-GNSS, 22-25 April, Graz, Austria

Khodabandeh A, Teunissen PJG (2014) Array-based satellite phase bias sensing: theory and GPS/BeiDou/QZSS results. Meas Sci and Technology 25, 095801

Knoop VL, de Bakker PF, Tiberius CCJM, van Arem B (2017) Lane determination with GPS precise point positioning. IEEE ITS Trans 18(9):2503-2513, doi:10.1109/TITS.2016.2632751

Lou Y, Zheng F, Gu S, Wang C, Guo H, Feng Y (2016) Multi-GNSS precise point positioning with raw singlefrequency and dual-frequency measurement models. GPS Solut 20(4):849-862

Marucci A, Colantoni A, Zambon I, Egidi G (2017) Precision farming in hilly areas: The use of network RTK in GNSS technology. Agriculture 7(60), doi:10.3390/agriculture7070060

Misra P, Enge P (2006) Global Positioning System: Signals, Measurements and Performance. Ganga-Jumana Press, Lincoln MA

Mongredien C, Doyen JP, Strom M, Ammann D (2016) Centimeter-Level Positioning for UAVs and Other Mass-Market Applications. In: ION GNSS, Portland, Oregon

Montenbruck O, Hauschild A, Steigenberger P, Hugentobler U, Teunissen PJG, Nakamura S (2013) Initial assessment of the COMPASS/BeiDou-2 regional navigation satellite system. GPS Solut 17(2):211-222 doi:10.1007/s10291-012-0272-x

Nadarajah N, Teunissen PJG, Raziq N (2013) BeiDou Inter-Satellite-Type Bias Evaluation and Calibration for Mixed Receiver Attitude Determination. Sensors 13(7):9435-9463

Odijk D (2002) Fast precise GPS positioning in the presence of ionospheric delays. PhD dissertation, Netherlands Geodetic Commission, Publications on Geodesy

Odijk D, Teunissen PJG (2008) ADOP in closed form for a hierarchy of multi-frequency single-baseline GNSS models. J Geod 82:473

Odijk D, Teunissen PJG (2013) Characterization of between-receiver GPS-Galileo inter-system biases and their effect on mixed ambiguity resolution. GPS Solut 17(4):521-533

Odolinski R, Teunissen PJG (2016) Single-frequency, dual-GNSS versus dual-frequency, single-GNSS: A low-cost and high-grade receivers GPS-BDS RTK analysis. J Geod 90(11):1255-1278

Odolinski R, Teunissen PJG (2017) Low-cost, highprecision, single-frequency GPS-BDS RTK positioning. GPS Solut doi:10.1007/s10291-017-0613-x

Odolinski R, Teunissen PJG, Odijk D (2015a) Combined BDS, Galileo, QZSS and GPS single-frequency RTK. GPS Solut 19(1):151-163, doi:10.1007/s10291-0140376-6

Odolinski R, Teunissen PJG, Odijk D (2015b) Combined GPS + BDS for short to long baseline RTK positioning. Meas Sci and Technology 26, 045801, doi: 10.1088/0957-0233/26/4/045801

Pan L, Zhang X, Liu J, Li X (2017) Performance evaluation of single-frequency precise point positioning with GPS, GLONASS, BeiDou and Galileo. J of Navigation 70(3):465-482

Paziewski J, Wielgosz P (2015) Accounting for GalileoGPS intersystem biases in precise satellite positioning. J Geod 89(1):81-93

Pesyna KM, Heath R, Humphreys TE (2014) Centimeter Positioning with a Smartphone-Quality GNSS Antenna. In: Proc. ION GNSS, Tampa, FL

Schaffrin B, Bock Y (1988) A unified scheme for processing GPS dual-band phase observations. Bull Geod 62:142-160

Schone T, Pandoe W, Mudita I, Roemer S, Illigner J, Zech C, Galas R (2011) GPS water level measurements for Indonesias tsunami early warning system. Nat Hazards Earth Syst Sci 11:741-749, doi:10.5194/nhess-11-7412011

Takasu T, Yasuda A (2008) Evaluation of RTK-GPS Performance with Low-cost Single-frequency GPS Receivers. In: International Symposium on GPS/GNSS, pp $852-861$

Teunissen PJG (1988) Towards a least-squares framework for adjusting and testing of both functional and stochastic model. Internal research memo Geodetic Computing Centre, Delft Reprint of original 1988 report (2004), No. 26

Teunissen PJG (1990a) An integrity and quality control procedure for use in multi sensor integration. In: Proceedings of the $3^{\text {rd }}$ International Technical Meeting of the Satelllite Division of the Institute of Navigation (ION GPS 1990), Colorado Spring, CO, pp 513-522, also published in: Volume VII of the GPS Red Book: Integrated systems, ION Navigation, 2012.

Teunissen PJG (1990b) GPS op afstand bekeken. In: Een halve eeuw in de goede richting Delft Lustrum book Snellius 215-233 
Teunissen PJG (1995) The least squares ambiguity decorrelation adjustment: a method for fast GPS integer estimation. J Geod 70: 65-82

Teunissen PJG (1997) A canonical theory for short GPS baselines. Part I: The baseline precision, Part II: The ambiguity precision and correlation, Part III: The geometry of the ambiguity search space, Part IV: Precision versus reliability. J Geod 71(6): 320-336, 71(7): 389-401, 71(8): 486-501, 71(9): 513-525

Teunissen PJG (1998a) Success probability of integer GPS ambiguity rounding and bootstrapping. J Geod 72:606612

Teunissen PJG (1998b) The Ionosphere-weighted GPS baseline precision in canonical form. J Geod 72: 107117

Teunissen PJG (1999) An optimality property of the integer least-squares estimator. J Geod 73:587-593

Teunissen PJG, Amiri-Simkooei AR (2008) Least-squares variance component estimation. J Geod 82(2):65-82

Teunissen PJG, Odolinski R, Odijk D (2014) Instantaneous BeiDou+GPS RTK positioning with high cut-off elevation angles. J Geod 88(4):335-350

Verhagen S, Teunissen PJG, Odijk D (2012) The Future of Single-Frequency Integer Ambiguity Resolution. In: N. Sneeuw et al. (eds.), VII Hotine-Marussi Symposium on Mathematical Geodesy, International Association of Geodesy Symposia 137, doi:10.1007/978-3-64222078-4_5

Wang X, Wenbo N (2016) An improved particle filter and its application to an INS/GPS integrated navigation system in a serious noisy scenario. Meas Sci and Technology 27, 095005

Wirola L, Alanen K, Kappi J, Syrjarinne J (2006) Bringing RTK to Cellular Terminals Using a Low-Cost Single-Frequency AGPS Receiver and Inertial Sensors. In: Position, Location, And Navigation Symposium, IEEE/ION, 25-27 April CA, USA

Wisniewski B, Bruniecki K, Moszynski M (2013) Eyaluation of RTKLIB's Positioning Accuracy Using low-cost GNSS Receiver and ASG-EUPOS. Int J on Marine Navigation and Safety of Sea Transportation 7(1):79-85

Zaminpardaz S, Teunissen PJG, Nadarajah N (2017) IRNSS/NavIC and GPS: a single- and dual-system L5 analysis. J Geod doi:10.1007/s00190-016-0996-4

Zhao S, Cui X, Guan F, Lu M (2014) A Kalman Filter-Based Short Baseline RTK Algorithm for SingleFrequency Combination of GPS and BDS. Sensors 14(8):15,415-15,433 